\title{
Announcement for co-contributing organization in previous article
}

\author{
Po-Chuan Hsieh
}

Received: 14 November 2010 / Accepted: 15 November 2010/Published online: 3 December 2010

(C) Springer-Verlag 2010

Dear Editor,

Our previous article, "The clinical experience of medulloblastoma treatment and the significance of time sequence for development of leptomeningeal metastasis", was published in Child Nervous System 2008 Dec; 24 (12): 1463-1467. Some efforts, especially data analysis, were contributed by colleagues in our associated institution. The full title of the contributing institutions should be: "Chang Gung Memorial Hospital, Chang Gang University and Medical College", and we would like to make this announcement for their efforts.

Best regards,

P.-C. Hsieh ( $₫)$

Department of Neurosurgery, Chang Gung Memorial Hospital,

Chang Gung University and Medical College,

No.5, Fu-Shin Street, Kwei-Shan,

Taoyuan 333 Taiwan, Republic of China

e-mail: PoChuan.Hsieh@gmail.com 